\title{
Tangence
}

\section{Lettres de Milady Juliette Catesby de Marie-Jeanne Riccoboni : lecture d'une délinquance}

Lucie Joubert

Numéro 47, mars 1995

Écritures au féminin : le genre marqué

URI : https://id.erudit.org/iderudit/025851ar

DOI : https://doi.org/10.7202/025851ar

Aller au sommaire du numéro

Éditeur(s)

Tangence

ISSN

0226-9554 (imprimé)

1710-0305 (numérique)

Découvrir la revue

Citer cet article

Joubert, L. (1995). Lettres de Milady Juliette Catesby de Marie-Jeanne Riccoboni : lecture d'une délinquance. Tangence, (47), 56-68.

https://doi.org/10.7202/025851ar d'utilisation que vous pouvez consulter en ligne.

https://apropos.erudit.org/fr/usagers/politique-dutilisation/ 


\section{Lettres de Milady Juliette Catesby de Marie- Jeanne Riccoboni : lecture d'une délinquance}

Lucie Joubert, Queen's University

Marie-Jeanne Riccoboni, actrice puis écrivaine du dixhuitième siècle français, que l'on a redécouverte il y a quelques années, consacra une grande partie de sa vie à la correspondance réelle et à la correspondance de fiction dans des romans dits épistolaires. Si Laclos, en épigraphe, plaça Les liaisons dangereuses sous le patronage de La nouvelle Héloïse ${ }^{1}$, Madame Riccoboni ne put bénéficier d'une telle source d'inspiration ni subir cette influence. Selon Joan Hinde Stewart, cette femme avant-gardiste avait devancé le grand Rousseau de plusieurs façons: d'abord, par la forme épistolaire qu'elle adopta dans ses romans Lettres de Fanni Butlerd et Lettres de Milady Juliette Catesby ${ }^{2}$, avant même que ce genre ne devienne, dans le sillage de La nouvelle Héloüse, le principal outil de fiction de la seconde moitié du siècle; par le contenu de ses romans, ensuite, qui fait d'elle une des rares praticiennes du "roman d'analyse" 3 .

Bien sûr, on pourrait répliquer que Madame Riccoboni, malgré le caractère novateur de son œuvre, s'inscrivait tout de même dans le prolongement d'une tradition épistolaire qui avait déjà atteint un haut niveau de raffinement dans l'expression, chez Madame de Sévigné, entre autres, et plusieurs de ses contemporains. Le glissement de la lettre réelle, c'est-à-dire cet instrument de communication entre deux individus, vers la lettre fictive, qui relève alors de la technique romanesque, bouleverse cependant les enjeux du "pacte d'intimité " 4 implicite entre les correspondants et en modifie les conditions de lecture.

1 René Pomeau, Laclos ou le paradoxe, Paris, Hachette, 1993, p. 133.

2 Le titre complet de l'ouvrage est le suivant: Lettres de Milady Juliette Catesby à Milady Henriette Campley, son amie. J'utilise la forme abrégée pour des raisons de commodité.

3 Joan Hinde Stewart, The Novels of Mme Riccoboni, Chapel Hill, University of North Carolina, coll. - Essays *, n' 8, 1976, p. 31.

4 L'expression est de Vincent Kaufman, dans L'équizoque épistolaire, Paris, Minuit, coll. $\cdot$ Critique $\cdot 1990$, p. 159. 
Dans un cadre fictionnel, en effet, nul besoin de supputer l'authenticité du propos ou l'intention du destinateur ${ }^{5}$; la mention générique efface dès l'abord la moindre ambiguité. Tout au plus pourrait-on convertir le mouvement de la lettre privée rendue publique en une intention de l'auteure de faire vrai au point de donner l'impression au lecteur qu'il s'est soudain introduit dans une réalité entre deux correspondants inconnus. Mais puisque c'est là, au fond, le désir - même partiel - de toute fiction, il faut plutôt s'attarder aux conséquences_de ce virage dans le contrat de lecture, à savoir le rôle du lecteur.

À la relation destinateur/destinataire qui prévaut dans la correspondance se substitue un nouveau type de dichotomie, ellemême à deux dimensions : d'une part, il y a les correspondants fictifs à l'intérieur de l'œuvre qui se lisent mutuellement et se répondent; d'autre part, le lecteur — "le tiers" — et, par un mouvement circulaire assez ironique, l'auteure, qui retrouve soudain sa nécessité dans le contexte du roman épistolaire, après avoir vu son importance estompée par les récentes théories narratologiques et leur parti pris pour un narrateur comme instance d'énonciation. Le fait de proposer au public une correspondance inventée cristallise en effet la présence de Madame Riccoboni et lève l'équivoque sur l'instance qui, prosaïquement, "fait parler" les personnages.

Cette transparence de l'intention éclipse donc le rôle de la narratrice pour réactualiser celui de l'auteure, phénomène que l'on peut observer fréquemment dans l'écriture au féminin. L'affirmation de soi, dans la littérature contemporaine des femmes plus particulièrement, a résisté à la fameuse "mort de l'auteur", selon une expression consacrée où le masculin prend tout son sens. Madame Riccoboni, à cet égard et loin des débats narratologiques, invite à une lecture neuve d'une société donnée; la fiction ici sert de filtre dans la mesure où elle permet d'échapper au piège restrictif du roman "à clés" sans toutefois gommer une caractéristique essentielle de l'ouvrage: le regard féminin qui se

5 Cette question, fort intéressante par atlleurs, a été longuement débattue dans les pages de cette revue; voir Daphni Baudoin (dir.), -Authenticité et littérature personnelle -, Tangence, $\mathrm{n}^{\circ} 45$, octobre 1994 et plus particulièrement le texte de Manon Brunet - La réalité de la fausse lettre. (p. 26-49) qui approfondit encore davantage la réflexion sur l'honnêteté de l'épistolier, son intention esthétique souvent virtuelle, et le lectorat qui s'immisce dans un processus privé. 
pose sur les gens et les choses ${ }^{6}$ et dont l'option de généricité même commande l'orientation de la lecture.

\section{Traditions et innovations}

On lit avec plaisir les passions mises en scène par Madame Riccoboni : un plaisir décuplé peut-être par le sentiment que des circonstances sociologiques et littéraires différentes auraient pu laisser dans l'oubli de tels testaments. La tentation de succomber au charme de l'exotisme de cette voix au féminin venue d'un autre siècle comporte toutefois quelques dangers; on risque plus aisément, à cause de l'écart temporel, de confondre tradition et innovation.

Ainsi pourrait-on se méprendre sur la question de l'identité. L'auteure française, au nom à consonance italienne, rédige, dans un procédé récurrent, des correspondances fictives qui prennent place dans la haute société britannique: Milady Juliette Catesby, dans le cas présent, voyage de Winchester au château d'Hastings et évoque Paris comme une lointaine contrée. Il serait fort mal venu de faire un lien entre ces identités éclatées et une quelconque recherche de soi, élément qui caractérise pourtant un grand nombre de textes féminins contemporains; l'histoire littéraire regorge d'exemples apatrides du genre et Madame Riccoboni, en optant pour ce procédé, cédait simplement à une mode de l'époque qui popularisait les us et coutumes britanniques ${ }^{7}$.

La quête d'une identité se trouve bien en deça de ces évidents repères géographiques, habilement camouflée entre les lignes de la correspondance et les anecdotes successives d'une passion qui renaîtra de ses cendres. Après avoir été abandonnée, pour d'obscures raisons, par l'amant qu'elle comptait épouser et qui s'est marié à une autre, Milady Juliette Catesby, dévastée, doit littéralement se recomposer pour survivre; à travers sa correspon-

6 L'arbitraire apparent de ce parti pris de lecture puise sa source dans une opinion avancée par plusieurs théoriciennes québécoises. Témoin Lori SaintMartin: - Les ouvres féminines sont déterminées par une culture des femmes en tant qu'expérience collective différente de celle des hommes et [...] elles doivent être lues ainsi *, Malaise et révolte des femmes dans la littérature qué bécoise depuis 1945, Québec, Les Cahiers de recherche du GREMF, 1989, p. 31.

7 Joan Hinde Stewart, op. cit., P. 32. 
dance assidue avec son amie Henriette, elle tentera de se redéfinir dans un jeu complexe de comparaisons-supputations né d'une observation précise et lucide de son entourage.

Les lettres constituent des réflexions, dans les deux sens du terme, qui donnent à voir une galerie de personnages féminins soupesés, évalués, par une femme trahie qui cherche la recette du détachement, l'attitude à adopter pour ne plus souffrir. "Je cherche à m'occuper des autres pour éloigner les idées qui me ramènent à moi-même" ${ }^{8}$, dit-elle. La fuite hors de soi n'est pas aisée; l'image obsédante de l'infidèle s'impose à son esprit malgré sa volonté. Cependant, la force psychologique du personnagé réside dans sa capacité à s'extirper, momentanément du moins, du marasme émotif pour porter attention à autrui et, d'une certaine façon, opposer une résistance à la douleur morale. Cette attitude contraste alors avec une idée courante au siècle précédent stipulant que la femme, de par sa "féminité", est naturellement disposée à écrire sur l'amour et surtout à s'abîmer dans les inévitables souffrances que lui occasionnera la passion? ${ }^{9}$.

Ces séquences dérivatives que ménage Madame Riccoboni à son héroïne permettent justement à l'auteure de "construire" le caractère de Juliette, c'est-à-dire de mieux le définir pour le lecteur qui voit alors s'élaborer sa pensée profonde. La lettre IV, par exemple, est presque entièrement consacrée à Lady Howard; l'apparente superficialité de l'amorce du personnage ne doit pas tromper:

Lady Howard est une très petite femme, assez jolie, point coquette, trop négligée même: elle conduit sa maison, [...] traite sérieusement les moindres détails et se fait une grande affaire de la plus petite chose. [...]

Il serait singulier que cette ménagère eût plus de mérite que nous: il est au moins bien sûr qu'elle a plus de bonheur. [...] son âme est sans cesse ouverte à l'impression du plaisir... Quel

8 Marie-Jeanne Riccoboni, Lettres de Milady Juliette Catesby. Roman, Paris, Desjonquères, 1983, p. 11. Dorénavant, les références à cet ouvrage seront indiquées dans le texte après la citation.

9 Voir l'intéressant article de Katherine A. Jensen, - Male Models of Feminine Epistolary; or, How to Write like a Woman in Seventeenth-Century France :, Elizabeth C. Goldsmith (dir.), Writing The Female Voice. Essays on Epistolary Literature, Boston, Northeastern University Press, 1989, p. 25-45, dans lequel elle identifie les caractéristiques de la - féminité - telles que vues par certains auteurs du dix-septième siècle qui écrivaient des lettres fictives signées de noms de femmes, entretenant ainsi un tenace malentendu. 
60

plaisir, me direz-vous? Eh ma chère Henriette, il en est de tant de sortes! une longue étude de nous-mêmes, notre raison, nos connaissances nous rendent-elles plus heureuses? Je ne sais quelle idée les autres peuvent avoir de cette lumière qu'on nomme esprit; elle se peint à mon imagination comme un flambeau ardent, qu'un coup de vent vient de souffler; il luit un peu dans l'ombre et ne la dissipe qu'à demi: sa faible clarté suffit pour montrer qu'on marche sur les bords d'un précipice mais non pas pour faire apercevoir l'endroit glissant où le pied peut manquer. On tombe, ma chère; et quand on a roulé jusqu'au fond, on a l'avantage de réfléchir et de se dire, tout froissé de sa chute, que si on avait mieux vu, on ne serait pas là. (p. 14-16)

Ce large extrait illustre le fonctionnement des réflexions de Juliette Catesby; une observation sur une femme donnée conduit au développement d'une pensée qui déborde vite le cadre de l'individuel pour rejoindre le collectif. Une telle ouverture permet alors de relativiser un drame intérieur personnel pour aborder une question plus générale. Ici, Juliette relance le débat rendu célèbre, par Molière entre autres, de l'accès des femmes à la connaissance. La déconfiture de la narratrice donne à lire l'échec ou à tout le moins le peu d'utilité de l'entreprise; constatation déprimante, s'il en est, d'une femme trop blessée dans ses espoirs amoureux pour évaluer sur l'heure le privilège d'être cultivée.

L'intérêt du doute soulevé par Madame Riccoboni chez Juliette réside cependant dans le fait que l'exercice s'effectue de l'intérieur. Il n'y a pas mise en scène d'une savante ridicule donnée en pâture à un public qui aura tôt fait de juger celle qui prétend en savoir autant que l'homme; on assiste plutôt à l'exposition des interrogations d'une femme intelligente, absolument dépourvue de complaisance envers elle-même, déçue par la vie et obligée de mettre en opposition ses connaissances et sa passion plutôt que de fusionner ces deux aspects fondamentaux de son existence. À quoi peut bien servir tout ce bagage intellectuel, toute cette lucidité,. s'ils ne permettent pas au moins d'espérer le bonheur? La franchise de Juliette trouvera sûrement écho chez la lectrice contemporaine qui, dans une conjoncture totalement différente entraînant d'autres conséquences, ne s'enl trouve pas moins devant un dilemme assez similaire.

Madame Riccoboni a aussi, dans l'ensemble de son ouvre et plus manifestement dans le roman qui nous intéresse, renversé 
l'idée reçue de la rivalité entre femmes ${ }^{10}$; le crêpage de chignons folklorique fait place à une amitié indéfectible entre Juliette, la narratrice, et sa fidèle destinataire Henriette. La relation entre les deux femmes, exemple avant l'heure de la solidarité féminine recherchée par les féministes, est empreinte d'une grande franchise et écarte la moindre coquetterie. Juliette peut ainsi dévoiler à sa correspondante des états d'âme extrêmes qu'elle cache au reste de l'entourage.

La lettre est bien sûr un exutoire à la déroute intérieure; la formulation des confidences au moment de crises émotives aiguës met en relief la profondeur de la confiance accordée à Henriette. "Mon Dieu, est-ce votre amie, est-ce une femme sensée, qui est si peu d'accord avec elle-même? Ma bonne, ma tendre amie, aimezmoi pour nous deux, car je me hais bien fort. (p. 86). Dans les luttes intimes qu'elle se livre à elle-même, dans sa résistance et son entêtement à ne pas consentir à revoir l'amant infidèle, Juliette constitue son unique ennemie. Vaincue par moments, elle remet littéralement son être entre les mains de sa correspondante qui se charge alors de réparer la situation. Juliette écrit ainsi, dans une lettre à Milord Carlile, après la rupture avec Milord d'Ossery: "Cette aimable fille me ramena insensiblement à moi-même. (p. 74).

Présence discrète - en partie à cause de la construction du roman sur laquelle on reviendra - mais constante, Henriette écoute, console, gronde doucement mais ne juge pas; ce personnage féminin d'une indéfectible loyauté, récurrent chez Riccoboni ${ }^{11}$, se. trouve, paradoxalement, à la fois éloigné et profondément impliqué dans le conflit intérieur de Juliette. Loin par la distance évidente qui sépare les correspondantes, mais aussi tenue à l'écart symboliquement par la nécessité romanesque d'assumer le rôle extérieur de "planche de salut" à laquelle se raccroche Juliette, Henriette maintient l'équilibre de son amie. "Je le hais... Je me hais... Je vous aime toujours. (p. 112), écrit encore Juliette. Cette constance dans les sentiments d'amitié se cristallisera à la toute fin du roman; mariée depuis peu à Milord d'Ossery, la narratrice réitère son pacte de fidélité: « Point de fêtes, de bals sans ma chère Henriette; je dirais point de plaisirs si la personne qui suit ma plume des yeux n'était déjà un peu

10 Janet Todd, Women's Friendship in Literature, New York, Columbia University Press, 1980, p. 353.

11 Janet Todd, op. cit. 
62

jalouse de ma tendre amitié (p. 174-175). Ainsi, le besoin d'une relation intime et privilégiée avec une femme ne s'incline pas, dans ce roman, devant l'abandon amoureux.

Outre les ressources de l'amitié féminine, Madame Riccoboni explore les méandres de la condition des femmes de son époque; selon Arlette André, "son féminisme vise à modifier le comportement de la femme, plutôt qu'à changer les structures sociales. [...] On découvre [...] fréquemment les flambées d'une révolte purement verbale, qui tourne court " ${ }^{12}$. Ce constat peut en effet synthétiser l'ensemble des renoncements auxquels sont contraintes les héroïnes de Madame Riccoboni ${ }^{13}$. Juliette Catesby, cependant, au cours de l'intrigue romanesque, doit au contraire renoncer $a$ renoncer à son amant; les chemins de l'expression de ses convictions féministes trouveront donc d'autres sentiers...

La narratrice exprimera en effet un profond désir d'égalité en des termes non équivoques dans une lettre à son ami Milord Carlile: "Dans cette route difficile où nous voyageons ensemble, le ciel nous a placés sur la même ligne; je puis marcher votre égale et je n'admets point de distinctions entre des créatures qui sentent, pensent et agissent de même (p. 46). L'allusion métaphorique à Dièu indique bien que l'assurance de l'égalité entre les sexes émane d'une instance incontestable. En même temps constat et profession de foi, cette affirmation trouve écho ailleurs, dans certaines observations, encore bien contemporaines toutes proportions gardées, des différences fondamentales entre les hommes et les femmes:

Heureux hommes, combien la différence de l'éducation, les préjugés, l'usage donnent d'avantage à ce sexe hardi qui ne rougit de rien, dit et fait tout ce qu'il veut. [...] Bas quand il désire, fier dès qu'il espère, ingrat lorsqu'il obtient... (p. 29)

12 Arlette André, - Le féminisme chez Madame Riccoboni-, Studies on Voltaire and The Eighteenth Century, Oxford, The Voltaire Foundation at the Taylor Institution, $1980, \mathrm{n}^{\circ} 193$, p. 1993.

13 Voir plus en détails ce même article d'Arlette André qui - fixe les limites de ce féminisme., p. 1993. Les exemples de l'auteure, à mon avis, bien que pertinents, me semblent évacuer la question du contexte social du xvir siècle. Le féminisme de Madame Riccoboni, ne s'inscrivant pas dans un long processus collectif - comme ce fut le cas dans notre siècle -, était une démarche personnelle donc à rayonnement réduit. Il n'est pas étonnant alors de découvrir, dans son ceuvre, une foule de personnages jouant le rôle d'opposants pour battre en brèche toute velléité de pouvoir. 
La lucidité ne facilite pas la vie de Juliette, loin de là. La révélation du motif de la fuite de son amant - dans un moment d'égarement, il a séduit une pauvre fille, l'a mise enceinte et l'a épousée pour la sauver du déshonneur et du suicide dont elle le menaçait - l'amène à s'exprimer une fois de plus sur le fossé qui sépare les sexes:

leur cour et leurs sens peuvent agir séparément; ils le prétendent au moins et par ces distinctions qu'ils prennent pour excuse, ils se réservent la faculté d'être excités par l'amour, séduits par la volupté ou entraînés par l'instinct. [...] Mais cette excuse qu'ils prennent, ils ne la reçoivent pas; remarquez cela : ce qu'ils séparent en eux, ils le réunissent en nous. C'est nous accorder une grande supériorité dans notre façon de sentir, mais faire naître en nous une terrible incertitude sur l'espèce des mouvements qui les portent à désirer de nous posséder. (p. 167-168)

Deux poids, deux mesures dans cette citation qui porte encore une fois les marques de ce "nous" collectif dont il a été question plus haut. La clairvoyance de la narratrice et ses doutes sur la nature des sentiments qu'elle inspire à l'homme ne l'empêcheront pas de l'épouser. Cette victoire du cœur sur la raison a fait des sceptiques parmi les analystes de Riccoboni; Joan Hinde Stewart émet, non sans ironie, des réserves quant à l'intelligence de cette union: "Juliette peut bien épouser Ossery et atteindre un certain épanouissement, mais il subsiste un fort doute que cette femme délicate et introspective pourra trouver un partenaire authentique dans le type d'homme qu'est Ossery ${ }^{14}$.

Une telle réticence est sans doute motivée, outre la perception générale qu'on a du personnage, par les dernières pages du roman. Milord d'Ossery écrit à Henriette: «Elle est à moi, pour jamais à moi! Plus de Milady Catesby" (p. 173). Le compliment a plutôt la consonance d'un constat de décès; la Juliette d'av'ant Ossery a disparu. La nouvelle épousée aurait-elle senti le danger pour répliquer ainsi, aussitôt: "En vérité on m'a mariée si vite', si vite, que je crois de bonne foi que le mariage ne vaut rien" (p. 173-174)?

14 Joan Hinde Stewart, op. cit., p. 119 (je traduis très librement). 
64

\section{Huis clos et ironie}

Madame Riccoboni a privilégié, dans sa construction romanesque, une correspondance à "sens unique". Les lettres proviennent exclusivement de Juliette Catesby et sont destinées à la fidèle Henriette ${ }^{15}$. Si le procédé se révèle coûteux dans l'étude d'une relation épistolaire authentique en ce qu'il occulte le point du vue du destinataire, il fournit au contraire dans un cadre fictif une plus grande latitude d'inteprétation: au lieu de se substituer à la narratrice ${ }^{16}$, le lecteur tend à s'identifier à la destinataire et à deviner le contenu des lettres auxquelles il n'a pas accès. La tâche lui est facilitée, toutefois, par l'emploi récurrent de l'italique qui indiquent la reprise d'un propos antérieur: "Vous craignez que vos lettres ne soient longues, qu'elles me fatiguent, vous, ma chère Henriette, penser que vous pouvez me fatiguer! (p. 84)

Cette astuce typographique sert d'autres effets comme la possibilité de répondre à un tiers par Henriette interposée: "me voilà à cinquante milles de Londres et je ne suis point morte: assurezen Milord Carlile. Malgré ses prédictions, je ne me suis point évanouie au pied du premier bêtre. (p. 9). Ou encore, par ce moyen, Juliette se cite elle-même, sous l'impulsion du moment, conférant au texte un caractère de parfaite synchronicité: "[Sir Henry] est près, tout près de moi... il lit presque ce que j'écris... je voudrais qu'il le lût pour lui apprendre... je continue exprès... Milord, pardon, vous permettez... Il s'incline, soupire et reste " (p. 25). L'italique, enfin, permet au lecteur de présumer de la totale honnêteté de l'épistolaire dans ses références à autrui en désignant, dans une substitution du' discours direct par l'indirect, les paroles prononcées textuellement: "[Sir Henry] a remarqué de longs soupirs qui m'échappent; il se doute qu'il y a un secret à une de mes bottes [...] Il est fort étonné que je vous écrive tous les jours" (p. 13).

Le lecteur constitue en principe le dernier maillon de la chaîne de réception. Force est de constater, pourtant, que cette

15 Seules exceptions : deux lettres à la toute fin, de Milord d'Ossery et de Milady d'Ormond, adressées à Henriette, toujours; Stewart explique cette entorse à la structure générale par la volonté de traduire la joyeuse confusion des jours entourant le mariage et de signifier que d'Ossery fait maintenant partie de la vie et de la correspondance de Juliette. Voir Joan Hinde Stewart, op. cit., p. 47-48.

16 Voir Manon Brunet, loc. cit., p. 30-31. 
correspondance assidue donne l'impression d'un huis clos au féminin jalousement gardé. Ainsi, lorsque la narratrice écrit à une tierce personne, Milord Carlile en l'occurrence, elle enchâsse cette missive dans une lettre à Henriette, faisant de son amie le témoin de ses moindres mouvements épistolaires. Les étapes de ses retrouvailles avec Milord d'Ossery, que l'on suit grâce à une correspondance entre les deux amants, obéiront au même procédé, comme si la réconciliation définitive ne pouvait se faire sans le consentement de la discrète Henriette.

L'insertion des lettres extérieures au strict échange entre Juliette et son amie n'est pas gratuite; elle sert en fait d'amorce à la narratrice pour une autocritique sans complaisance qu'elle tient à effectuer devant témoin. Après avoir recopié une lettre assez dure qu'elle destinait à Milord d'Ossery, Juliette se relit et se juge: "Ne trouvez-vous pas, ma chère Henriette, une espèce de fausseté dans cette façon d'écrire [...] N'eút-il pas été mieux [...] d'avouer ma véritable situation à son égard; de dire Je vous aime peut-être encore, mais je ne vous estime plus, je renonce à vous" (p. 103).

On remarque au passage une nouvelle utilisation de l'italique qui cette fois exprime le "non-écrit". Il faut aussi s'arrêter, pour paraphraser Juliette, sur ces "façons d'écrire "; Madame Riccoboni, en effet, a su ménager des styles différents pour chacun des intervenants de la correspondance "recopiée" par l'héroïne. Le style de la Comtesse de Sunderland, par exemple, est plus altier, plus sobre, sans les exclamations qui scandent le discours de la narratrice, et évite toute spontanéité; l'ensemble n'est pourtant pas froid et suscite une vague admiration chez Juliette qui, sans partager ses idées, s'incline tout de même devant tant de contrôle de soi.

L'auteure fait de ce style une affaire de personnalité et d'identité: dans la lettre XI, Juliette remarque: "Ah ma chère Henriette, vous aimez Milord Carlile bien plus que vous ne le croyez! Vous prenez son style sans vous en apercevoir" (p. 39). La longue lettre explicative de Milord d'Ossery est pour sa part bâtie comme un exercice de rhétorique visant à prouver non pas son innocence mais sa bonne foi et fait contraste avec les emportements de Juliette; la narration détaillée des événements qui ont jadis mené à la rupture renoue avec une linéarité plus traditionnelle qui s'était estompée dans une correspondance pourtant tenue quotidiennement. Le discours rationnel de l'homme, ici, explique 
66

pour convaincre: l'opération est une réussite, semble-t-il, puisque l'amante blessée pardonne. Madame Riccoboni, en poussant ainsi son héroïne dans les bras de d'Ossery, n'en trahit pas pour autant son pacte de complicité féminine : le plaidoyer de l'amant suscite d'abord chez Juliette une sympathie... pour Jenny, l'épouse défunte, qu'elle plaint d'avoir été si malheureuse malgré le mariage qui la sauva du déshonneur!

La communication épistolaire implique une distance entre les deux correspondants, cela va de soi; cette même distance devient, chez Madame Riccoboni, propice à l'expression d'une certaine ironie, c'est-à-dire à un recul vis-à-vis des gens, des choses mais surtout de soi-même. Juliette, par auteure interposée, manie les outils rhétoriques avec dextérité pour créer des effets savoureux, comme ces mots antithétiques qui, par leur juxtaposition, donnent à lire un piquant contraste: "Enchantée de son nouvel état, [une veuve qui n'a pas tout à fait vingt ans] vient ici passer l'année de son deuil, seulement pour méditer en repos sur le choix qu'elle fera, lorsque la bienséance lui permettra de remplacer un vieux qu'elle haïssait de tout son cœur" (p. 17).

La finesse de la formulation et le secret - fallacieux, il est vrai - de cette correspondance incite, dirait-on, le lecteur à l'indulgence face à des commentaires railleurs: *Belle en tout point, belle depuis le matin jusqu'au soir [...l Si on lui adresse la parole, elle est si persuadée qu'on va lui faire un compliment, qu'un signe de remerciement précède toujours son attention" (p. 27). Méchante sans doute, cette observation n'en démontre pas moins la perspicacité d'une spectatrice qui fouille les âmes pour les démasquer. Madame Riccoboni maîtrise aussi bien l'art du sous-entendu: la périphrase "l'époux de Jenny Monfort" (p. 92) sous la plume de Juliette suffit à rendre le sentiment de profonde trahison qui anime la narratrice; de même, l'expéditive gradation contenue dans la courte phrase *Vous mourriez d'envie que Sir Henry parlât; eh bien, le voilà déclaré, proposé et refusé! * (p. 113) traduit la célérité avec laquelle elle a réglé le cas du malheureux soupirant...

$\mathrm{Au}$-delà de l'anecdote, il faut cependant noter un resserrement significatif des notes ironiques à mesure que Juliette voit se rapprocher l'éventualité, à peine concevable quelques jours auparavant, d'une réconciliation avec Milord d'Ossery. Les remarques auto-ironiques, c'est-à-dire celles que Juliette se destine à ellemême, témoignent de sa lucidité en toutes circonstances: ainsi, 
toute à la préparation du mariage de Sara, elle écrit: "Je suis gaie, ma chère; je commence à reprendre le goût des amusements; je ne veux pas examiner la cause de ce changement, je trouverais peut-être... N'allez pas croire que le mariage de Sara soit un prétexte pour célébrer la convalescence de ce pauvre Comte..." (p. 129). Donnant elle-même les clés d'interprétation de son antiphrase, Juliette, en se moquant de ses faux-semblants, reste fidèle au contrat de transparence qui la lie à Henriette. Tout comme dans cet autre exemple, qui, sur un mode sentencieux, annonce le dénouement à venir: "Je suis fâchée d'avoir envoyé cette lettre : on dit qu'entre des amants brouillés un reproche est le préliminaire d'un traité de paix" (p. 134).

Si Juliette se refuse à la complaisance, elle ne ménage pas non plus l'objet de ses tourments. Les commentaires se font d'ailleurs plus cinglants à mesure qu'elle se sent sur le point de faiblir et de consentir au retour de l'amant prodigue, comme si, par la distance artificielle de l'ironie, elle cherchait à creuser l'écart entre elle et celui auquel elle peut de moins en moins résister. Assurée du rétablissement d'Ossery, elle se moque allègrement de la maladie qui vient pourtant de lui coûter bien des inquiétudes :

Oh, ces hommes! ces hommes! Remarquez-vous comment ils savent tirer parti des événements: lorsque les moyens de nous subjuguer semblent leur manquer un incident imprévu, un hasard, une maladie les ramènent vers le but qu'ils s'étaient proposé. [...] Quand ils ne savent plus que faire, ils ont la fièvre, ma chère, ils n'ont plus qu'un instant à vivre [...] Ses torts sont-ils diminués? Non, mais... il a été malade. (p. 125-126)

Plus loin, après avoir lu la longue confession d'Ossery, elle réplique, reproduisant par l'italique le discours qui raconte "le * moment d'égarement : "quelle aventure! ce cabinet... cette obscurité... sa hardiesse... il appelle cela un malbeur... J'oubliai mon amour, dit-il...Ah, oui, les hommes ont de ces oublis" (p. 167). La force même de cette ironie du désespoir pratiquée surtout dans les dernières lettres trahit le combat intérieur: Juliette ne peut se leurrer longtemps sur la nature de ses sentiments et le rempart qu'elle croyait avoir érigé entre eux s'effrite aussitôt...

L'ironie dans cette correspondance constitue une forme de délinquance par rapport au bon usage. Madame Riccoboni n'en est pas à une infraction près: ses écrits témoignent d'une liberté de pensée et d'une audace romanesque qui lui ont valu bien des 
68

critiques ${ }^{17}$. Ses essais en traduction confirment, si besoin est, sa soif d'organiser à sa guise le monde et le texte, dût-il appartenir à un autre ${ }^{18}$. Son ouvre est l'expression d'une intelligence autonome qui a anticipé à maints égards la pensée contemporaine et traversé les siècles pour venir se greffer sans mal aux réflexions actuelles des femmes.

17 Certaines sont d'ailleurs bien méritées. Stewart écrit, au sujet de la lettre XX: - Juliette continue de se demander, pendant qu'elle compose une page complète, ce que peut bien lui vouloir d'Ossery, s'exclamant "je ne peux écrire" alors qu'elle continue à le faire. (op. cit., p. 49, je traduis librement).

18 Voir à ce sujet Stewart, op. cit., p. 32-34. 